Proyecciones

Vol. 23, $\mathrm{N}^{o}$ 1, pp. 61-72, May 2004.

Universidad Católica del Norte

Antofagasta - Chile

\title{
ORLICZ - PETTIS THEOREMS FOR MULTIPLIER CONVERGENT OPERATOR VALUED SERIES
}

\author{
CHARLES SWARTZ \\ New Mexico State University, USA \\ Received November 2003. Accepted March 2004.
}

\begin{abstract}
Let $X, Y$ be locally convex spaces and $L(X, Y)$ the space of continuous linear operators from $X$ into $Y$. We consider 2 types of multiplier convergent theorems for a series $\sum T_{k}$ in $L(X, Y)$. First, if $\lambda$ is a scalar sequence space, we say that the series $\sum T_{k}$ is $\lambda$ multiplier convergent for a locally convex topology $\tau$ on $L(X, Y)$ if the series $\sum t_{k} T_{k}$ is $\tau$ convergent for every $t=\left\{t_{k}\right\} \in \lambda$. We establish conditions on $\lambda$ which guarantee that a $\lambda$ multiplier convergent series in the weak or strong operator topology is $\lambda$ multiplier convergent in the topology of uniform convergence on the bounded subsets of X. Second, we consider vector valued multipliers. If $E$ is a sequence space of $X$ valued sequences, the series $\sum T_{k}$ is $E$ multiplier convergent in a locally convex topology $\eta$ on $Y$ if the series $\sum T_{k} x_{k}$ is $\eta$ convergent for every $x=\left\{x_{k}\right\} \in E$. We consider a gliding hump property on $E$ which guarantees that a series $\sum T_{k}$ which is $E$ multiplier convergent for the weak topology of $Y$ is $E$ multiplier convergent for the strong topology of $Y$.
\end{abstract}




\section{INTRODUCTION}

- The original Orlicz-Pettis Theorem which asserts that a series $\sum x_{k}$ in a normed space $X$ which is subseries convergent in the weak topology of $X$ is actually subseries convergent in the norm topology of $X$ can be interpreted as a theorem about multiplier convergent series. Let $\lambda$ be a scalar sequence space which contains the space of all sequences which are eventually 0 and let $(E, \tau)$ be a Hausdorff topological vector space. The series $\sum x_{k}$ in $E$ is $\lambda$ multiplier convergent with respect to $\tau$ if the series $\sum t_{k} x_{k}$ is $\tau$ convergent for every $t=\left\{t_{k}\right\} \in \lambda$; the elements of $\lambda$ are called multipliers. Multiplier convergent series where the multipliers come from some of the classical sequence spaces, such as $l^{p}$, have been considered by various authors $([\mathrm{B}],[\mathrm{FP}])$; in particular, $c_{0}$ multiplier convergent series have been used to characterize Banach spaces which contain no copy of $c_{0}([\mathrm{D}])$. Thus, a series $\sum x_{k}$ is subseries convergent if and only if $\sum x_{k}$ is $m_{0}$ multiplier convergent, where $m_{0}$ is the sequence space of all scalar sequences which have finite range. Several Orlicz-Pettis Theorems have been established for multiplier convergent series where the multipliers are from various classical sequence spaces ([LCC], [SS],[WL]). In this note we consider Orlicz-Pettis Theorems for multiplier convergent series of linear operators where the multipliers are both scalar and vector valued. In general, as Example 2.1 below shows, a series of operators which is $m_{0}$ multiplier convergent in the weak operator topology is not $m_{0}$ multiplier convergent in the topology of uni-

form convergence on bounded subsets; however, if the space of multipliers satisfies certain conditions an Orlicz-Pettis result of this type does hold. One of our results contains a gliding hump assumption on the multiplier space which is of independent interest and also yields an Orlicz-Pettis result for series in the strong topology of a general locally convex space. In the final section we use the gliding hump property introduced in section 2 to establish a result on the weak sequential completeness of $\beta$-duals similar to a result of Stuart ([S1],[S2]).

We begin by fixing the notation and terminology which we use. Let $X, Y$ be real Hausdorff locally convex spaces and let $L(X, Y)$ be the space of continuous linear operators from $X$ into $Y$. If $x \in X, y^{\prime} \in Y^{\prime}$, let $x \otimes y^{\prime}$ be the linear functional on $L(X, Y)$ defined by $\left\langle x \otimes y^{\prime}, T\right\rangle=\left\langle y^{\prime}, T x\right\rangle$ and let $X \otimes Y^{\prime}$ be the linear subspace spanned by $\left\{x \otimes y^{\prime}: x \in X, y^{\prime} \in Y^{\prime}\right\}$. The weak operator topology on $L(X, Y)$ is the weak topology from the duality between $L(X, Y)$ and $X \otimes Y^{\prime}$ ([DS]VI.1). The strong operator topology on $L(X, Y)$ is the topology of pointwise convergence on $X$ ([DS]VI.1). Let 
$L_{b}(X, Y)$ be $L(X, Y)$ with the topology of uniform convergence on the bounded subsets of $X$; the topology of $L_{b}(X, Y)$ is generated by the seminorms $p_{A}(T)=\sup \{p(T x): x \in A\}$, where $p$ is a continuous semi-norm on $Y$ and $A$ is a bounded subset of $X$ ([DS],VI.1).

\section{SCALAR MULTIPLIERS}

. In this section we establish Orlicz-Pettis type theorems for multiplier convergent series of operators with respect to the weak operator topology and the topology of $L_{b}(X, Y)$. As the following example illustrates, an operator valued series which is subseries or $m_{0}$ multiplier convergent in the strong (weak) operator topology needn't be $m_{0}$ multiplier convergent in $L_{b}(X, Y)$.

Example 1. Define $T_{k}: l^{1} \rightarrow l^{1}$ by $T_{k} t=\left\langle e^{k}, t\right\rangle=t_{k} e^{k}$, where $e^{k}$ is the sequence with 1 in the $k t h$ coordinate and 0 in the other coordinates.. Since for every subsequence $\left\{n_{k}\right\}$ the series $\sum_{k=1}^{\infty} T_{n_{k}} t=\sum_{k=1}^{\infty} t_{n_{k}} e^{n_{k}}$ converges in $l^{1}$ for every $t \in L^{1}$, the series $\sum T_{k}$ is $m_{0}$ multiplier convergent in the strong operator topology. But, $\left\|T_{k}\right\|=1$ so $\sum T_{k}$ is not $m_{0}$ multiplier convergent in the operator norm.

However, as the following two theorems show, if the space of multipliers $\lambda$ satisfies some additional conditions, an operator valued series which is $\lambda$ multiplier convergent in the weak(strong) operator topology can indeed be $\lambda$ multiplier convergent in $L_{b}(X, Y)$.

The sequence space $\lambda$ is an AK space if $\lambda$ has a Hausdorff locally convex topology such that the coordinate maps $t=\left\{t_{k}\right\} \rightarrow t_{k}$ from $\lambda$ to $\mathbf{R}$ are continuous and each $t$ has a series expansion $t=\sum_{k=1}^{\infty} t_{k} e^{k}$ which converges in the topology of $\lambda([\mathrm{BL}])$.

Theorem 2. If $\lambda$ is a barrelled AK space and $\sum T_{k}$ is $\lambda$ multiplier convergent in the weak operator topology of $L(X, Y)$, then $\sum T_{k}$ is $\lambda$ multiplier convergent in $L_{b}(X, Y)$.

Proof: From Corollary 2.4 of [SS] it follows that $\sum T_{k}$ is $\lambda$ multiplier convergent in the strong topology $\beta\left(L(X, Y), X \otimes Y^{\prime}\right)$. Thus, it suffices to show that if a sequence(net) $\left\{S_{k}\right\}$ in $L(X, Y)$ converges in $\beta\left(L(X, Y), X \otimes Y^{\prime}\right)$, then $\left\{S_{k}\right\}$ converges in $L_{b}(X, Y)$; that is, the strong topology $\beta(L(X, Y), X \otimes$ $\left.Y^{\prime}\right)$ is stronger than the topology of $L_{b}(X, Y)$. Let $A \subset X$ be bounded, $B \subset$ $Y^{\prime}$ be equicontinuous and let $C=\left\{x \otimes y^{\prime}: x \in A, y^{\prime} \in B\right\}$. It is easily 
checked that $C$ is $\sigma\left(X \otimes Y^{\prime}, L(X, Y)\right)$ bounded so $\sup \left\{\left|\left\langle x \otimes y^{\prime}, S_{k}\right\rangle\right|: x \in\right.$ $\left.A, y^{\prime} \in B\right\} \rightarrow 0$ which implies that $S_{k} x \rightarrow 0$ uniformly for $x \in A$ or $S_{k} \rightarrow 0$ in $L_{b}(X, Y)$.

We next consider an analogue of Theorem 2 for other spaces of multipliers which are described by purely algebraic conditions in contrast to the AK and barrelledness assumptions of Theorem 2 .

Let $E$ be a vector space of $X$ valued sequences which contains the space of all $X$ valued sequences which are eventually 0 . If $t=\left\{t_{j}\right\}$ is a scalar sequence and $x=\left\{x_{j}\right\}$ is either a scalar or $X$ valued sequence, $t x=\left\{t_{j} x_{j}\right\}$ will be the coordinatewise product of $t$ and $x$. A sequence of interval $\left\{I_{j}\right\}$ in $\mathbf{N}$ is increasing if $\max I_{j}<\min I_{j+1}$ for all $j$; if $I$ is an interval, then $C_{I}$ will be the characteristic function of $I$. The space $E$ has the infinite gliding hump property ( $\infty$-GHP) if whenever $x \in E$ and $\left\{I_{k}\right\}$ is an increasing sequence of intervals, there exist a subsequence $\left\{n_{k}\right\}$ and $a_{n_{k}}>0, a_{n_{k}} \rightarrow \infty$ such that every subsequence of $\left\{n_{k}\right\}$ has a further subsequence $\left\{p_{k}\right\}$ such that $\sum_{k=1}^{\infty} a_{p_{k}} C_{I_{p_{k}}} x \in E$ (coordinate sum). [The term $\infty$-GHP is used to suggest that the "humps" $C_{I} x$ are multiplied by a sequence which tends to $\infty$; there are other gliding hump properties where the "humps" are multiplied by elements of classical sequence spaces $([\mathrm{Sw} 3])]$.

We now give several examples of spaces with the $\infty$-GHP. The space $E$ is normal (solid in the scalar case) if $l^{\infty} E=E$ ([KG]2.1); $E$ is $c_{0}$-invariant if $x \in E$ implies that there exist $t \in c_{0}, y \in E$ such that $x=t y$ ([G]; the term $c_{0}$ - factorable might be more descriptive).

Example 3. If $E$ is normal and is $c_{0}$-invariant, then $E$ has $\infty$-GHP. Let $x \in E$ with $x=t y$ where $t \in c_{0}, y \in E$ and let $\left\{I_{k}\right\}$ be an increasing sequence of intervals. Pick an increasing sequence $\left\{n_{k}\right\}$ such that $\sup \left\{\left|t_{j}\right|\right.$ : $\left.j \in I_{n_{k}}\right\}=b_{n_{k}}>0$ (if this choice is impossible there is nothing to do). Note that $b_{n_{k}} \rightarrow 0$ so $a_{n_{k}}=1 / b_{n_{k}} \rightarrow \infty$. Define $v_{j}=t_{j} a_{n_{k}}$ if $j \in I_{n_{k}}$ and $v_{j}=0$ otherwise; then $v \in l^{\infty}$ so $v y \in E$ since $E$ is normal. We have $\sum_{j=1}^{\infty}(v y)_{j} e^{j}=\sum_{k=1}^{\infty} a_{n_{k}} C_{I_{n_{k}}} x \in E$. Since the same argument can be applied to any subsequence of $\left\{n_{k}\right\}, E$ has $\infty$-GHP.

We now give some examples of spaces which satisfy the conditions of Example 3.

Example 4. Let $X$ be a normed space and let $c_{0}(X)$ be the space of all null sequences in $X$. Then $c_{0}(X)$ is normal and $c_{0}$-invariant and so has $\infty$-GHP.. 
Example 5. Let $0<p<\infty$ and let $X$ be a normed space. Let $l^{p}(X)$ be the space of all $p^{\text {th }}$ power summable sequences in $X$. Let $x \in l^{p}(X)$ and pick an increasing sequence $\left\{n_{k}\right\}$ such that $\sum_{j=n_{k}+1}^{n_{k+1}}\left\|x_{j}\right\|^{p}<1 / 2^{k(p+1)}$. Set $I_{k}=\left[n_{k}+1, n_{k+1}\right], t=\sum_{k=1}^{\infty} 2^{-k} C_{I_{k}}, y=\sum_{k=1}^{\infty} 2^{k} C_{I_{k}} x$ so $x=t y$ with $t \in c_{0}$ and $y \in l^{p}$. Hence, $l^{p}(X)$ is $c_{0}$-invariant and is obviously normal.

Likewise, it is easily checked that the spaces $d=\left\{t: \sup _{k}\left|t_{k}\right|^{1 / k}<\infty\right\}$ and $\delta=\left\{t: \lim \left|t_{k}\right|^{1 / k}=0\right\}$ (see $[\mathrm{KG}]$ p.48 and 68) are normal and $c_{0}-$ invariant and, hence, have $\infty-G H P$.

There exist non-normal sequence spaces with $\infty$-GHP.

Example 6. Let $c s$ be the space of convergent series ([KG]). Let $t \in c s$ and $\left\{I_{k}\right\}$ be an increasing sequence of intervals. Choose a subsequence $\left\{n_{k}\right\}$ such that $\left|\sum_{j \in I_{n_{k}} \cap I} t_{j}\right|<1 / k 2^{k}$ for any interval $I$. Consider $s=$ $\sum_{k=1}^{\infty} k C_{I_{n_{k}}} t$. If $I$ is any interval contained in the interval [ $\left.\min I_{n_{k}}, \infty\right)$, then

$$
\left|\sum_{j \in I} s_{j}\right|=\left|\sum_{i=k}^{\infty} \sum_{j \in I_{n_{i}} \cap I} i t_{j}\right| \leq \sum_{i=k}^{\infty} 1 / 2^{i}=2^{-k+1}
$$

so the partial sums of the series generated by $s$ are Cauchy and $s \in c s$. Since the same argument applies to any subsequence of $\left\{n_{k}\right\}$, cs has $\infty$ GHP.

Note that the argument above shows that any Banach $A K$ space has $\infty$-GHP;e.g., $b v_{0}([\mathrm{KG}])$.

The spaces $l^{\infty}, m_{0}, b s$ and $b v$ do not have $\infty$-GHP.

We next consider a result analogous to Theorem 2 except that we use the strong operator topology.

Theorem 7. Let $\lambda$ have $\infty$-GHP. If $\sum T_{j}$ is $\lambda$ multiplier convergent in the strong operator topology, then $\sum T_{j}$ is $\lambda$ multiplier convergent in $L_{b}(X, Y)$.

Proof: If the conclusion fails, there exist $\varepsilon>0, t \in \lambda, A \subset X$ bounded, a continuous semi-norm $p$ on $Y$ and subsequences $\left\{m_{k}\right\},\left\{n_{k}\right\}$ such that $m_{1}<n_{1}<m_{2}<\ldots$ and $p_{A}\left(\sum_{l=m_{k}}^{n_{k}} t_{l} T_{l}\right)>\epsilon$. For every $k$ there exists $x_{k} \in A$ such that

(1) $p\left(\sum_{l=m_{k}}^{n_{k}} t_{l} T_{l} x_{k}\right)>\varepsilon$. 
Set $I_{k}=\left[m_{k}, n_{k}\right]$. Since $\lambda$ has $\infty$-GHP, there exist $\left\{p_{k}\right\}, a_{p_{k}}>0, a_{p_{k}} \rightarrow$ $\infty$ such that every subsequence of $\left\{p_{k}\right\}$ has a further subsequence $\left\{q_{k}\right\}$ such that $s=\sum_{k=1}^{\infty} a_{q_{k}} C_{I_{q_{k}}} t \in \lambda$. Let $M=\left[m_{i j}\right]=\left[\sum_{l=m_{j}}^{n_{j}}\left(t_{l} a_{p_{j}}\right) T_{l}\left(x_{i} / a_{p_{i}}\right)\right]$. We use the Antosik-Mikusinski Matrix Theorem ([Sw2 ]2.2.2) to show that the diagonal of $M$ converges to 0 ; this will contradict (1). First, the columns of $M$ converge to 0 since $x_{i} / a_{p_{i}} \rightarrow 0$ and each $T_{l}$ is continuous. Next, given a subsequence there is a further subsequence $\left\{q_{k}\right\}$ such that $s=\sum_{k=1}^{\infty} a_{q_{k}} C_{I_{q_{k}}} t \in \lambda$. The series $\sum_{l=1}^{\infty} s_{l} T_{l}$ converges in the strong operator topology to an operator $T \in L(X, Y)$. Hence, $\sum_{j=1}^{\infty} m_{i q_{j}}=$ $\sum_{j=1}^{\infty} \sum_{l \in I_{q_{j}}} s_{l} T_{l}\left(x_{i} / a_{p_{i}}\right)=T\left(x_{i} / a_{p_{i}}\right) \rightarrow 0$. It follows that $M$ is a $K$ matrix. By the Antosik-Mikusinski Matrix Theorem the diagonal of $M$ converges to 0 which contradicts (1).

Remark 8. If the multiplier space $\lambda$ in Theorem 7 is normal, we may replace the assumption that $\sum T_{j}$ is $\lambda$ multiplier convergent in the strong operator topology with the assumption that the series is $\lambda$ multiplier convergent in the weak operator topology. For if $\lambda$ is normal and $\sum t_{j} T_{j}$ is convergent in the weak operator topology for every $t \in \lambda$, then the series is subseries convergent in the weak operator topology and, therefore, convergent in the strong operator topology by the classical Orlicz-Pettis Theorem.

The proof of Theorem 7 also establishes the following version of the Orlicz-Pettis Theorem for multiplier convergent series which should be compared to the version given in Corollary 2.4 of [SS] where it is assumed that lamda is a barrelled AK-space.

Theorem 9.(Orlicz-Pettis) If $\lambda$ has $\infty$-GHP and if $\sum_{j} x_{j}$ is $\lambda$ multiplier convergent in the weak topology $\sigma\left(X, X^{\prime}\right)$, then $\sum_{j} x_{j}$ is $\lambda$ multiplier convergent in the strong topology $\beta\left(X, X^{\prime}\right)$.

The spaces $d=\left\{t: \sup _{k}\left|t_{k}\right|^{1 / k}<\infty\right\}$ and $\delta=\left\{t: \lim \left|t_{k}\right|^{1 / k}=0\right\}$ ([KG]) give examples of spaces to which Theorems 7 and 9 apply but to which Theorem 2 and its scalar counterpart, Corollary 2.4 of [SS], do not apply. (The natural metric on $d$ does not give a vector topology $([\mathrm{KG}] \mathrm{p}$. 68).)

The scalar versions of Theorems 2 and 7 are both of interest. That is, the case when the space $Y$ is the scalar field. 
Corollary 10. Assume that $\lambda$ has $\infty-G H P$ or that $\lambda$ is a barrelled AK space.

If $\sum_{j} x_{j}^{\prime}$ is $\lambda$ multiplier convergent in the weak* topology $\sigma\left(X^{\prime}, X\right)$ of $X^{\prime}$, then $\sum_{j} x_{j}^{\prime}$ is $\lambda$ multiplier convergent in the strong topology $\beta\left(X^{\prime}, X\right)$.

Corollary 10 should be compared to the Diestel-Faires Theorem concerning subseries convergence in the weak* topology of the dual of a Banach space. In the Diestel-Faires result the emphasis is on conditions on the space while in Corollary 10 the conditions are on the space of multipliers.

\section{VECTOR MULTIPLIERS}

. We now consider vector valued multipliers for operator valued series. Let $\left\{T_{k}\right\} \subset L(X, Y)$ and let $E$ be a vector space of $X$ valued sequences containing the space of all sequences which are eventually 0 . If $\tau$ is a locally convex Hausdorff topology on $Y$, we say that the series $\sum T_{k}$ is $E$ multiplier convergent with respect to $\tau$ if the series $\sum T_{k} x_{k}$ is $\tau$ convergent for every $x=\left\{x_{k}\right\} \in E$. We establish Orlicz-Pettis Theorems for multiplier convergent series analogous to Theorems 2 and 7 for the weak and strong topologies of $Y$.

As in section 2 the following example shows that if the space of multipliers does not satisfy some condition an Orlicz-Pettis Theorem does not in general hold for the weak and strong topologies of $Y$.

Example 1. Let $l^{\infty}(X)$ be the space of all bounded $X$ valued sequences. Assume that $l^{\infty}$ has the weak topology $\sigma\left(l^{\infty}, l^{1}\right)$ Define $P_{k}$ : $l^{\infty} \rightarrow l^{\infty}$ by $P_{k} x=x_{k} e^{k}$. Let $E=l^{\infty}\left(l^{\infty}\right)$. If $x=\left\{x^{k}\right\} \in E$, then $\sum P_{k} x^{k}=\sum x_{k}^{k} e^{k}$ is $\sigma\left(l^{\infty}, l^{1}\right)$ convergent, but if $x=\left\{e^{k}\right\} \in E$, then $\sum P_{k} e^{k}=\sum e^{k}$ is not $\beta\left(l^{\infty}, l^{1}\right)=\|\|_{\infty}$ convergent.

We begin with the analogue of Theorem 2.7 since it is straightforward to state and prove.

Theorem 2. Let $E$ have $\infty$-GHP and $\left\{T_{k}\right\} \subset L(X, Y)$. If $\sum T_{k}$ is $E$ multiplier convergent with respect to the weak topology $\sigma\left(Y, Y^{\prime}\right)$, then $\sum T_{k}$ is $E$ multiplier convergent with respect to the strong topology $\beta\left(Y, Y^{\prime}\right)$.

Proof: If the conclusion fails, there exist $x \in E,\left\{y_{k}^{\prime}\right\} \sigma\left(Y^{\prime}, Y\right)$ bounded, $\varepsilon>0$ and subsequences $\left\{m_{k}\right\},\left\{n_{k}\right\}$ with $m_{1}<n_{1}<m_{2}<\ldots$ and 
$\left|\sum_{l=m_{k}}^{n_{k}}\left\langle y_{k}^{\prime}, T_{l} x_{l}\right\rangle\right|>\varepsilon$ for all $k$. Set $I_{k}=\left[m_{k}, n_{k}\right]$. Since $E$ has $\infty$-GHP, there exist $\left\{p_{k}\right\}, a_{p_{k}}>0, a_{p_{k}} \rightarrow \infty$ such that every subsequence of $\left\{p_{k}\right\}$ has a further subsequence $\left\{q_{k}\right\}$ such that $\sum a_{q_{k}} C_{I_{q_{k}}} x \in E$. Define an infinite $\operatorname{matrix} M=\left[m_{i j}\right]=\left[\sum_{l \in I_{p_{j}}}\left\langle y_{p_{i}}^{\prime} / a_{p_{i}}, T_{l}\left(a_{p_{j}} x_{l}\right)\right\rangle\right]$. We show that $M$ is a $K$ matrix so the diagonal of $M$ converges to 0 by the Antosik-Mikusinski Matrix Theorem ([Sw2]2.2.2) and this will contradict the inequality above. First, the columns of $M$ converge to 0 since $\left\{y_{i}^{\prime}\right\}$ is $\sigma\left(Y^{\prime}, Y\right)$ bounded and $a_{p_{i}} \rightarrow \infty$. Next, given a subsequence there is a further subsequence $\left\{q_{j}\right\}$ such that $y=\sum a_{q_{k}} C_{I_{q_{k}}} x \in E$. Let $\sum_{l=1}^{\infty} T_{l} y_{l}=\sum_{j=1}^{\infty} \sum_{l \in I_{q_{j}}} T_{l}\left(a_{q_{j}} x_{l}\right)=z$ be the $\sigma\left(Y, Y^{\prime}\right)$ sum of this series. Then $\sum_{j=1}^{\infty} m_{i q_{j}}=\left\langle y_{q_{i}}^{\prime} / a_{q_{i}}, z\right\rangle \rightarrow 0$ so $M$ is a $K$ matrix and the result follows.

We next establish the analogue of Theorem 2.2. If $u \in X, e^{k} \otimes u$ is the sequence with $u$ in the $k^{t h}$ coordinate and 0 in the other coordinates. If $\tau$ is a Hausdorff locally convex topology on $E,(E, \tau)$, or $E$ if $\tau$ is understood, is an AK space if $x=\tau-\lim _{n} \sum_{k=1}^{n} e^{k} \otimes x_{k}=\sum_{k=1}^{\infty} e^{k} \otimes x_{k}$ for every $x \in E$ $([\mathrm{BL}])$.

Let $\sum T_{k}$ be $E$ multiplier convergent with respect to $\sigma\left(Y, Y^{\prime}\right)$. Define a linear map $\widehat{T}: E \rightarrow Y$ by setting $\widehat{T} x=\sigma\left(Y, Y^{\prime}\right)-\lim _{n} \sum_{k=1}^{n} T_{k} x_{k}=$ $\sum_{k=1}^{\infty} T_{k} x_{k}$. Note that $\widehat{T}\left(e^{k} \otimes u\right)=T_{k} u$ for $u \in X, k \in \mathbf{N}$. Recall that the (scalar) $\beta$-dual of $E$ is defined to be $E^{\beta}=\left\{\left\{y_{i}^{\prime}\right\}: \sum_{i=1}^{\infty}\left\langle y_{i}^{\prime}, x_{i}\right\rangle\right.$ converges $\forall x \in E\}([\mathrm{BL}])$. If $y^{\prime}=\left\{y_{i}^{\prime}\right\} \in E^{\beta}$ and $x=\left\{x_{i}\right\} \in E$, we set $y^{\prime} \cdot x=$ $\sum_{i=1}^{\infty}\left\langle y_{i}^{\prime}, x_{i}\right\rangle$ and note that $E$ and $E^{\beta}$ are in duality with respect this bilinear pairing. If $y^{\prime} \in Y^{\prime}, x \in E$, then $\left\langle y^{\prime}, \widehat{T} x\right\rangle=\left\langle y^{\prime}, \sum T_{k} x_{k}\right\rangle=\sum\left\langle y^{\prime}, T_{k} x_{k}\right\rangle=$ $\sum\left\langle T_{k}^{\prime} y^{\prime}, x_{k}\right\rangle$ so $\left\{T_{k}^{\prime} y^{\prime}\right\} \in E^{\beta}$ and $\left\langle y^{\prime}, \widehat{T} x\right\rangle=\left\{T_{k}^{\prime} y^{\prime}\right\} \cdot x$. Hence, $\widehat{T}$ is $\sigma\left(E, E^{\beta}\right)-\sigma\left(Y, Y^{\prime}\right)$ continuous and, therefore, $\beta\left(E, E^{\beta}\right)-\beta\left(Y, Y^{\prime}\right)$ continuous $([\mathrm{W}] 11.2 .3,[\mathrm{Sw} 1] 26.15)$ so we have

Theorem 3. If $\left(E, \beta\left(E, E^{\beta}\right)\right)$ is an $\mathrm{AK}$ space and $\sum T_{k}$ is $E$ multiplier convergent with respect to $\sigma\left(Y, Y^{\prime}\right)$, then $\sum T_{k}$ is $E$ multiplier convergent with respect to $\beta\left(Y, Y^{\prime}\right)$.

Proof: If $x \in E, \widehat{T} x=\widehat{T}\left(\sum e^{k} \otimes x_{k}\right)=\beta\left(Y, Y^{\prime}\right)-\lim _{n} \sum_{k=1}^{n} \widehat{T}\left(e^{k} \otimes x_{k}\right)=$ $\beta\left(Y, Y^{\prime}\right)-\lim _{n} \sum_{k=1}^{n} T_{k} x_{k}$ by the strong continuity of $\widehat{T}$ established above.

If $E$ is a barrelled AK space, then the argument in Lemma 3.9 of $[\mathrm{KG}]$ shows that $E^{\prime}=E^{\beta}$ and since $E$ is barreled, the original topology of $E$ 
is just $\beta\left(E, E^{\beta}\right)$ so Theorem 3 is applicable. If $X$ is a Banach space, the spaces $c_{0}(X)$ of null sequences with the sup-norm and $l^{p}(X)$ of absolutely psummable sequences with the $l^{p}$-norm give examples of barrelled (B-spaces), AK spaces to which Theorem 3 applies.

\section{WEAK SEQUENTIAL COMPLETENESS OF}

$\beta$-DUALS. The infinite gliding hump property introduced in section 2 also has applications to the $\beta$-duals of vector valued sequence spaces. In particular, we use one of the results to establish the weak sequential completeness of $\beta$-duals which serves as a complement to a weak sequential completeness result of Stuart ([S1],[S2]).

The $\beta$-dual of $E$ with respect to $Y, E^{\beta Y}$, us the space of all sequences $\left\{T_{j}\right\} \subset L(X, Y)$ such that the series $\sum T_{j}$ is $E$ multiplier convergent ([BL]). Our main result in this section concerns uniform convergence of series for elements in $\beta$-duals.

Theorem 1. Assume that $E$ has $\infty$-GHP. If $\Gamma \subset E^{\beta Y}$ is pointwise bounded on $E$ with respect to $\beta\left(Y, Y^{\prime}\right)$, then for every $x \in E$ the series $\sum_{k} T_{k} x_{k}$ are uniformly $\beta\left(Y, Y^{\prime}\right)$ convergent for $T \in \Gamma$.

Proof: If the conclusion fails, there exist $\epsilon>0,\left\{T_{k}\right\} \subset \Gamma,\left\{y_{k}^{\prime}\right\} \subset Y^{\prime}$ $\sigma\left(Y^{\prime}, Y\right)$ bounded and subsequences $\left\{m_{k}\right\},\left\{n_{k}\right\}$ with $m_{1}<n_{1}<m_{2}<\ldots$ and

( 2 ) $\left|\left\langle y_{k}^{\prime}, \sum_{l=m_{k}}^{n_{k}} T_{l} x_{l}\right\rangle\right|>\epsilon$.

Set $I_{k}=\left[m_{k}, n_{k}\right]$. By $\infty$-GHP, there exist $\left\{p_{k}\right\}, a_{p_{k}}>0, a_{p_{k}} \rightarrow \infty$ such that every subsequence of $\left\{p_{k}\right\}$ has a further subsequence $\left\{q_{k}\right\}$ such that $\sum a_{q_{k}} C_{I_{q_{k}}} \in E$. Define an infinite matrix

$M=\left[m_{i j}\right]=\left[\left\langle y_{p_{i}}^{\prime} / a_{p_{i}}, \sum_{l=m_{p_{j}}}^{n_{p_{j}}} T_{l}^{p_{i}}\left(a_{p_{j}} x_{l}\right)\right\rangle\right]$. Since $\left\{T^{i}\right\}$ is pointwise $\beta\left(Y, Y^{\prime}\right)$ bounded on $E,\left\{y_{i}^{\prime}\right\}$ is $\sigma\left(Y^{\prime}, Y\right)$ bounded and $1 / a_{p_{i}} \rightarrow 0$, the columns of $M$ converge to 0 . Next, given any subsequence there is a further subsequence $\left\{q_{j}\right\}$ such that $u=\sum_{j=1}^{\infty} a_{q_{j}} C_{I_{q_{j}}} x \in E$. Therefore, $\sum_{j=1}^{\infty} m_{i q_{j}}=\left\langle y_{p_{i}}^{\prime} / a_{p_{i}}, \sum_{j=1}^{\infty} T^{p_{i}} u_{j}\right\rangle \rightarrow 0$ by the same argument as above. Hence, $M$ is a $K$ matrix so the diagonal of $M$ converges to 0 . This contra$\operatorname{dicts}(2)$.

Theorem 1 can be used to establish a weak sequential completeness result for the $\beta$-dual $E^{\beta Y}$. The weak topology, $\sigma\left(E^{\beta Y}, E\right)$, on $E^{\beta Y}$ from 
$E$ is the weakest topology on $E^{\beta Y}$ such that all of the maps $x \rightarrow \sum_{k} T_{k} x_{k}$ from $E$ into $Y$ are continuous for every $T \in E^{\beta Y}$. The pair $(X, Y)$ has the Banach-Steinhaus property if whenever $\left\{T_{k}\right\} \subset L(X, Y)$ is such that $\lim T_{k} x=T x$ exists for every $x \in X$, then $T \in L(X, Y)$. For example, if $X$ is barrelled, then $(X, Y)$ has the Banach-Steinhaus property ([W]9.3.7 , $[\mathrm{Sw}] 24.12$ ).

Lemma 2. If $\left\{T^{k}\right\} \subset E^{\beta Y}$ is $\sigma\left(E^{\beta Y}, E\right)$ Cauchy, for each $x \in E$ the series $\sum_{j} T_{j}^{k} x_{j}$ are uniformly convergent for $k \in \mathbf{N}$ and $(X, Y)$ has the Banach-Steinhaus property, then there exists $T \in E^{\beta Y}$ such that $T^{k} \rightarrow T$ with respect to $\sigma\left(E^{\beta Y}, E\right)$.

Proof: Suppose that $\left\{T^{k}\right\}$ is $\sigma\left(E^{\beta Y}, E\right)$ Cauchy. For each $j$ and $z \in X$ , $\lim _{k} T^{k}\left(e^{j} \otimes z\right)=T_{j}(z)$ exists. By the Banach-Steinhaus property $T_{j} \in$ $E^{\beta Y} ;$ put $T=\left\{T_{j}\right\}$.

We claim that $T \in L(X, Y)$ and $T^{k} \rightarrow T$ in $\sigma\left(E^{\beta Y}, E\right)$. Let $x \in E$. Put $u=\lim T^{k} x$. It suffices to show that $u=\sum_{j=1}^{\infty} T_{j} x_{j}$. Let $U$ be a balanced neighborhood of 0 in $Y$ and pick a balanced neighborhood of $0 \mathrm{~V}$ such that $V+V+V \subset U$. There exists $p$ such that $\sum_{j=n}^{\infty} T_{j}^{k} x_{j} \in V$ for $n \geq p, k \in \mathbf{N}$. Fix $n \geq p$. Pick $k_{n}=k$ such that $\sum_{j=1}^{\infty} T_{j}^{k} x_{j}-u \in V$ and $\sum_{j=1}^{n}\left(T_{j}^{k}-T_{j}\right) x_{j} \in V$. Then $\sum_{j=1}^{n} T_{j} x_{j}-u=\left(\sum_{j=1}^{\infty} T_{j}^{k} x_{j}-u\right)+\sum_{j=1}^{n}\left(T_{j}-\right.$ $\left.T_{j}^{k}\right) x_{j}-\sum_{j=1}^{\infty} T_{j}^{k} x_{j} \in V+V+V \subset U$ and the result follows.

Corollary 3. Assume that $E$ has $\infty$-GHP and $Y$ is barrelled. If $(X, Y)$ has the Banach-Steinhaus property,then

$\left(E^{\beta Y}, \sigma\left(E^{\beta Y}, E\right)\right)$ is sequentially complete.

Proof: Since $Y$ is barrelled, the original topology of $Y$ is $\sigma\left(E^{\beta Y}, E\right)$. The result then follows from Theorem 1 and Lemma 2.

Stuart has established a weak sequential completeness result similar the Corollary 3 but involving a different gliding hump condition call the signed gliding hump property (signedWGHP) ([S1],[S2]). The space $E$ has signedWGHP if whenever $x \in E$ and $\left\{I_{k}\right\}$ is an increasing sequence of intervals, there is a subsequence $\left\{n_{k}\right\}$ and a sequence of signs $\left\{s_{k}\right\}, s_{k}= \pm 1$ such that $\sum s_{k} C_{I_{n_{k}}} x \in E$ [coordinatewise sum]. The two gliding hump conditions, the $\infty$-GHP and the signedWGHP, are independent; the space $b s$ has signedWGHP but not $\infty$-GHP ([S1],[S2]) while the space $b v_{0}$ has $\infty$-GHP but not signedWGHP (remark following Example 2.6). Thus, Corollary 3 and Theorem 3.5 of [S2] cover different spaces of multipliers or different $\beta$-duals. 


\section{References}

[B] G. Bennett, Some inclusion theorems for sequence spaces, Pacific J. Math., 46, pp. 17-30, (1973).

[BL] J. Boos and T. Leiger, Some distinguished subspaces of domains of operator valued matrices, Results Math., 16, pp. 199-211, (1989).

[D] J. Diestel, Sequences and Series in Banach Spaces, Springer-Verlag, N. Y., (1984).

[DF] J. Diestel and B. Faires, Vector Measures, Trans. Amer. Math. Soc., 198, pp. 253-271, (1974).

[DS] N. Dunford and J. Schwartz, Linear Operators I, Interscience, N. Y., (1958).

[FP] M. Florencio and P. Paul, A note on $\lambda$ multiplier convergent series, Casopis Pro Pest. Mat., 113, pp. 421-428, (1988).

[G] D. J. H. Garling, The $\beta$ - and $\gamma$-duality of sequence spaces, Proc. Cambridge Phil. Soc., 63, pp. 963-981, (1967).

[KG] P. K. Kamthan and M. Gupta, Sequence Spaces and Series, Marcel Dekker, N. Y., (1981).

[LCC] Li Ronglu, Cui Changri and Min Hyung Cho, An invariant with respect to all admissible (X,X')-polar topologies, Chinese Ann. Math.,3, pp. 289-294, (1998).

[S1] C. Stuart, Weak Sequential Completeness in Sequence Spaces, Ph.D. Dissertation, New Mexico State University, (1993).

[S2] C. Stuart, Weak Sequential Completeness of $\beta$-Duals, Rocky Mountain Math. J., 26, pp. 1559-1568, (1996).

[SS] C. Stuart and C. Swartz, Orlicz-Pettis Theorems for Multiplier Convergent Series, Journal for Analysis and Appl.,17, pp. 805-811, (1998).

[Sw1] C. Swartz, An Introduction to Functional Analysis,Marcel Dekker, N. Y., (1992). 
[Sw2] C. Swartz, Infinite Matrices and the Gliding Hump, World Sci.Publ., Singapore, (1996).

[Sw3] C. Swartz, A multiplier gliding hump property for sequence spaces, Proy. Revista Mat., 20, pp. 19-31, (2001).

[W] A. Wilansky, Modern Methods in Topological Vector Spaces, McGraw-Hill, N. Y., (1978).

[WL] Wu Junde and Li Ronglu, Basic properties of locally convex Aspaces, Studia Sci. Math. Hungar., to appear.

\section{Charles Swartz}

Mathematics Department

New Mexico state University

Las Cruces, NM 88003

USA

e-mail : cswartz@nmsu.edu 